\title{
Siva is an apoptosis-selective p53 target gene important for neuronal cell death
}

\author{
SBR Jacobs ${ }^{1}$, S Basak ${ }^{1}$, JI Murray ${ }^{2,3}$, N Pathak ${ }^{1}$ and LD Attardi; ${ }^{\star, 1,2}$
}

p53 plays a central role in neuronal cell death resulting from acute injury or disease. To define the pathway by which p53 triggers apoptosis, we used microarray analysis to identify p53 target genes specifically upregulated during apoptosis but not cell cycle arrest. This analysis identified a small subset of targets highly selective for the p53 apoptotic response, including Siva, a proapoptotic protein whose function is not well understood. Siva's expression pattern suggests that it plays an instructive role in apoptosis, and accordingly, we demonstrate that Siva is essential for p53-dependent apoptosis in cerebellar granule neurons. In addition, we determine that endogenous Siva is associated with the plasma membrane and that Caspase-8 and Bid are important for neuronal apoptosis. Our studies highlight the participation of membrane signaling events in p53's apoptotic program in primary neurons and have significant implications for understanding the mechanisms underlying pathogenesis after neuronal injury and in neurodegenerative diseases.

Cell Death and Differentiation (2007) 14, 1374-1385; doi:10.1038/sj.cdd.4402128; published online 20 April 2007

Engagement of an apoptotic program by the p53 tumor suppressor is a beneficial organismal response toward combating neoplasia. ${ }^{1}$ In contrast, activation of p53-dependent cell death has deleterious consequences following neuronal damage. $^{2}$ Specifically, p53-dependent apoptosis is implicated in the pathogenesis ensuing from acute neuronal injury, such as following ischemic stroke, traumatic brain injury, and seizures, as well as in diseases of progressive neurodegeneration, including Alzheimer's, Parkinson's, and Huntington's. ${ }^{2-4}$ Increased levels of p53 protein, associated with apoptosis, are detected in brains of patients with these neurological disorders, consistent with findings that expression of p53 is sufficient to induce apoptosis in cultured neurons. ${ }^{4}$ Compelling evidence for the importance of p53 in pathological neuronal cell death in vivo comes from the analysis of mouse models of ischemic stroke or of Alzheimer's, Parkinson's, and Huntington's diseases, where p53-deficiency prevents neuronal apoptosis and rescues disease phenotypes. ${ }^{3,5-7}$ Given the crucial role of p53dependent cell death in neuronal disease and tumor suppression, it is important to unravel the mechanisms by which p53 triggers an apoptotic response.

p53 function as a transcriptional activator is important for its ability to induce apoptosis in response to certain cellular stresses, such as DNA damage. ${ }^{8,9}$ As a result, significant effort has been devoted to identifying p53 target genes that mediate its apoptotic responses, and several targets, includ- ing Perp (p53 effector of apoptosis related to Pmp22), Puma, Noxa, and Bax, have been shown to be important for p53dependent apoptosis in vivo. ${ }^{10-14}$ However, despite the known requirement for these gene products in p53-mediated apoptosis, the mechanism by which p53 directs an apoptotic response as opposed to other fates it can induce, such as cell cycle arrest, is unclear. For example, although genes such as Bax and Noxa are required for a maximal apoptotic response, they are induced to similar levels during p53-mediated G1 arrest and apoptosis, ${ }^{13,15}$ indicating that they are not responsible for determining whether cells undergo apoptosis. To better define the pathway through which p53 specifies a cell death program, we performed genomic analysis to identify genes selectively activated by p53 during apoptosis, which may dictate apoptotic cell fate. By comparing the expression profiles of apoptotic, arresting, and p53-deficient mouse embryonic fibroblasts (MEFs), we identified the proapoptotic gene Siva as an apoptosis-selective p53 target gene. Furthermore, we established that Siva encodes a membrane protein which, along with other extrinsic apoptotic pathway components, serves as a key mediator of p53-dependent apoptosis in primary neurons. These findings underscore the importance of membrane signaling for p53-dependent apoptosis in neurons and have great implications for understanding the p53-mediated cell death underlying neuronal injury and neurodegenerative diseases.

\footnotetext{
${ }^{1}$ Division of Radiation and Cancer Biology, Department of Radiation Oncology, Stanford University School of Medicine, Stanford, CA, USA and ${ }^{2}$ Department of Genetics, Stanford University School of Medicine, Stanford, CA, USA

*Corresponding author: LD Attardi, Departments of Radiation Oncology and Genetics, Stanford University School of Medicine, CCSR-South, Room 1255, 269 Campus Drive, Stanford, CA 94305-5152, USA. Tel: + 1650725 8424; Fax: + 1650723 7382; E-mail: attardi@ stanford.edu

${ }^{3}$ Current address: Department of Genomic Sciences, University of Washington, Seattle, WA 98195, USA.

Keywords: Siva; p53; apoptosis; neurons; microarray

Abbreviations: MEFs, mouse embryonic fibroblasts; E1A-MEFs, E1A-expressing MEFs; dChip, DNA-Chip analyzer; Perp, p53 effector of apoptosis related to Pmp22; Nm23-M4, expressed in non-metastatic cells 4; Daf-1, decay accelerating factor-1; Gtse, G two $S$ phase expressed protein 1; Apaf-1, apoptotic protease activating factor-1; CGN, cerebellar granule neurons; DIV, days in vitro; shRNAs, short hairpin RNAs; EGFR, epidermal growth factor receptor; TNF, tumor necrosis factor; S.E.M., standard error of the mean

Received 09.5.06; revised 07.12.06; accepted 31.1.07; Edited by V De Laurenzi; published online 20.4.07
} 


\section{Results}

A limited number of p53-dependent genes are selectively induced during apoptosis. To identify genes that may play an instructive role in the p53 apoptotic program, we undertook a genomic-level microarray analysis to identify p53 target genes selectively induced during apoptosis, utilizing a MEF system that affords the unique possibility to compare the p53 apoptotic and G1 arrest responses in parallel contexts. Whereas wild-type MEFs respond to DNA damage by activating a p53-dependent G1 checkpoint, MEFs expressing certain oncoproteins, such as adenovirus $\mathrm{E} 1 \mathrm{~A}$, respond to DNA damage by undergoing p53-dependent apoptosis. ${ }^{16,17}$ We compared the expression profiles of apoptotic E1A-p53+/+ MEFs to G1-arrested $p 53+/+$ MEFs and to E1A-p53-/- deficient MEFs to identify p53-dependent genes that are preferentially expressed during apoptosis (Figure 1a). We first examined global gene expression profiles by hierarchical clustering using average linkage analysis, which generated six clusters (Figure 1b). p53-activated genes, identified as genes with augmented expression in E1A-p53+/+ MEFs compared to E1A-p53-/- MEFs, are present in clusters iii and iv. Strikingly, only 78 of the 554 p53-dependent genes are in the cluster of genes whose expression is selectively upregulated during apoptosis (cluster iv, Figure $1 \mathrm{~b}$ and c), whereas 476 genes are expressed during both arrest and apoptosis (cluster iii, Figure 1b, Supplementary Figure 1). Importantly, the p53 target gene we previously identified as being highly expressed specifically during apoptosis, Perp, is present in cluster iv. ${ }^{15}$ In contrast, many known transcriptional targets of p53, including Bax, Noxa, p21, Mdm2 and Cyclin G1, are present in cluster iii, consistent with previous work showing that these genes are expressed during both p53-mediated arrest and apoptosis. ${ }^{13,15}$ These findings confirm the efficacy of our screen and reveal novel genes induced selectively during p53-dependent apoptosis.

In addition to hierarchical clustering, we utilized DNA-Chip analyzer (dChip) to identify genes induced in the apoptotic population compared to both the G1-arrested and E1A-p53deficient populations. Consistent with our clustering data, dChip analysis yields a dramatically small group of p53dependent, apoptosis-selective induced genes, with only 27 being identified out of the $\sim 12000$ genes and ESTs represented on the array (Table 1 ). Interestingly, we found very few genes whose expression selectively decreases during p53-dependent apoptosis (Figure 1b, Supplementary Figure 2, Table 2), suggesting that repression of gene expression is not a major mechanism specifying p53mediated apoptosis in DNA damage-treated MEFs.

The finding that 14 of 27 genes identified by dChip are also present in cluster iv supports the idea that they are bona fide differentially expressed genes. To definitively assess their apoptosis-selectivity, we performed Northern blot analysis on G1-arrested, apoptotic, and E1A-p53-deficient MEFs, focusing on a subset of genes identified by both Cluster and dChip (Figure 1d). All of the genes examined are indeed expressed to a greater extent during apoptosis than during G1 arrest, illustrating the power of our array analysis for identifying differentially expressed genes with potential roles in p53- dependent apoptosis. In fact, we identified genes previously established as direct transcriptional targets of p53, several of which have known associations with apoptosis, including Perp, Apaf-1 (apoptotic protease activating factor-1), and Siva. ${ }^{10,18,19}$ Interestingly, the proteins encoded by these genes do not clearly participate in one common biological function. For example, these proteins include Nm23-M4 (expressed in non-metastatic cells 4), a member of a family of mitochondrial nucleoside diphosphate kinases associated with cancer, ${ }^{20}$ Daf-1 (decay accelerating factor-1), an inhibitor of complement activation also linked to metastasis, ${ }^{21}$ and several metabolic genes, such as phosphomannomutase and diacylglycerol kinase-alpha. Thus, in our genome-wide analysis, we identified apoptosis-selective gene products with a variety of activities, suggesting the potential importance of diverse pathways for p53-dependent apoptosis. Understanding the functions of these proteins will illuminate mechanisms of apoptosis that may be cued in different cellular contexts.

Siva is an apoptosis-selective gene induced in various contexts of p53-mediated apoptosis. Among the p53 target genes identified by our array analysis as being highly expressed specifically during apoptosis, Siva, given its known apoptotic activity, ${ }^{19,22}$ stood out as a strong candidate effector of p53-dependent apoptosis. Siva's apoptosis-selective expression profile is distinct from that of other p53 proapoptotic target genes, such as Noxa, which is induced during both G1 arrest and apoptosis, in a pattern indistinguishable from the G1 arrest mediator p21 (Figure 2a). Further, the fact that Siva is not related to classical apoptosis regulators, such as proapoptotic Bcl-2 family members, suggested that analysis of Siva might provide new insight into mechanisms of p53-mediated apoptosis.

To define the role of Siva in the p53 apoptotic pathway, we first sought to determine if Siva induction occurs generally during p53-activated apoptosis by examining Siva levels in several additional apoptotic contexts. When wild-type and p53-/- MEFs were exposed to $20 \mathrm{~J} / \mathrm{m}^{2}$ UVC, we found Siva levels were augmented in wild-type cells, concomitant with the induction of p53-dependent apoptosis (Figure 2b). At later times following UVC, when MEFs begin to undergo p53independent apoptosis, Siva levels failed to increase in p53-/- MEFs, confirming that Siva induction is linked to p53-dependent apoptosis. The specificity of Siva upregulation to $p 53-d e p e n d e n t$ apoptosis was further established using $\mathrm{E} 1 \mathrm{~A} /$ Ras-expressing, p53-/- MEFs, which undergo p53-independent apoptosis upon tumor necrosis factor- $\alpha$ (TNF- $\alpha$ ) treatment, ${ }^{15}$ but do not show Siva induction (Figure $2 \mathrm{c}$ ). To establish if Siva is induced by p53 during apoptosis in other cell types, we utilized LTR6 cells, mouse myeloid leukemia cells expressing temperature-sensitive p53. ${ }^{23}$ Siva levels are greatly enhanced upon activation of p53 and induction of apoptosis in LTR6 cells (Figure 2d). Finally, we examined cerebellar granule neurons (CGN), where p53-dependent apoptosis occurs in response to DNA-damaging agents such as camptothecin (see Figure 4a and Enokido et al. ${ }^{24}$ ). Siva expression in CGN is induced by camptothecin in a p53-dependent manner (Figure 2e). Together, these data indicate that Siva is induced in diverse contexts of p53-dependent, but not p53-independent, 
apoptosis. In addition, consistent with a previous report showing that overexpressed p53 binds and activates transcription through a p53 consensus site in the first intron of Siva, ${ }^{22}$ we a

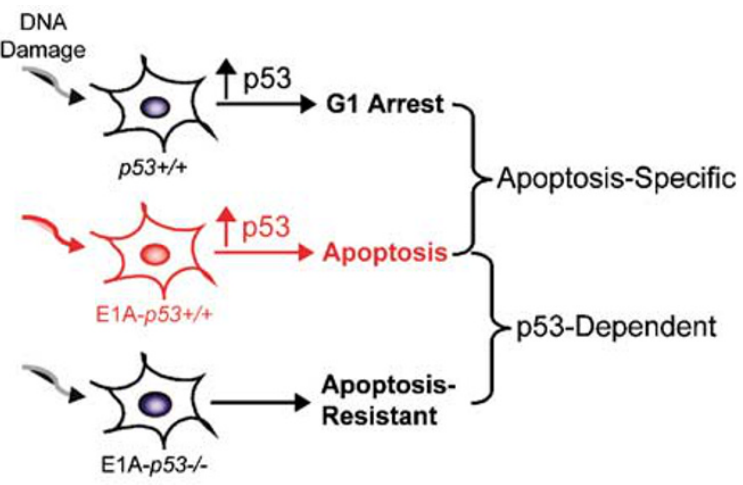

C

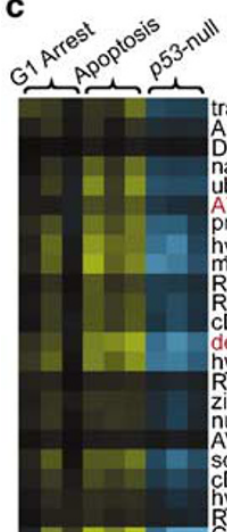
Ainase (PRKA) anchor protein 2 nanos homolog 1 (drosophila) ubiguitin-activating enzyme $1 \mathrm{C}$
ATP-binding cassette, sub-family $\mathrm{B}$ (MDR/TAP), member $1 \mathrm{~B}$
proteasome (prosome, macropaln) subunit, beta type 9 thetical mypothetical protein LOC212285 (AA718110 RIKEN CDNA 2410089813 gene (Al854360)
RIKEN CDNA 2900057 K09 gene (Al854413) CDNA sequence BC026370 (AA655098) decay accelerating factor 1 (Daf-1) hypothetical protein L0C212285 (AA718110) RIKEN CDNA 2310069104 gene (Al850676) . 81937

(ute carrier family 14 (urea transporter), member 1 DNA sequence BCO28881 (AW124729) (A) ransmembrane protein with EGF-like and two follistatin-like domains 1 DNA segment, Chr 2. Brigham and Women 's Genetics 1356 expressed RlKEN CDNAAI30092 J06 gene (AA739339) RIKEN cDNA 1110028N05 gene (AI854291) cerebral cavernous malformations 1 G two S phase expressed protein 1 (Gtse/B99) CD27 Binding Protein (Siva) p53 effector of apoptosis related to Pmp22 (Perp) AV 338260 phosphatidylinositol glycan, class Mus musculus 13 days embryo foreblimb cDNA (A1847298) cytochrome c. oxidase subunit Vlb, testes-specific (AW123567) embryonic ectoderm development utrophin

(AW227 ring finger protein 14 diacylglycerc
AV2 33314 early growth response protein 1 adenosine kinase AW012491 hosphorylase kinase gamma RIKEN CDNA D030041N15 gene (AW050141) RIKEN CDNA 1110008 K06 gene (AW049897) promyelocytic leukemia transmembrane 7 superfamily member 3

core binding factor beta RIKEN CDNA $2810407 \mathrm{~K} 09$ gene (AA98579 Bernardelli-Seip congenital lipodystrophy 2 homolog (human) transportin 2 (importin 3, karyopherin beta $2 \mathrm{~b}$ ) Al848713 RIKEN CDNA 1110064N10 gene (AW124599) (ubiquinione) 1 , alpha/beta subcomplex programmed cell death 6 interacting protein (human) homeobox D12 U40494

expressed sequence Al834976

issue inhibitor of metalloproteinase

RIKEN cDNA 2410004 C24 gene (Al849500) DNA segment, Chr 10, ERATO Doi 214 expressed CC76551 RIKEN CDNA 1810004l06 gene (Al839743) AV 321418 (Asp-Glu-Ala-Asp) box polypeptide 25 A kinase (PRKA) anchor protein 1 DnaJ (Hsp40) homolog, subfamily B, member 5 AV 312779 RIKEN CDNA 2010107K23 gene (AA710297) mus musculus transcribed sequences (Al839289) found that endogenous p53 from apoptotic MEFs binds this element (Supplementary Figure 3), further supporting the idea that Siva is a direct transcriptional target of p53.

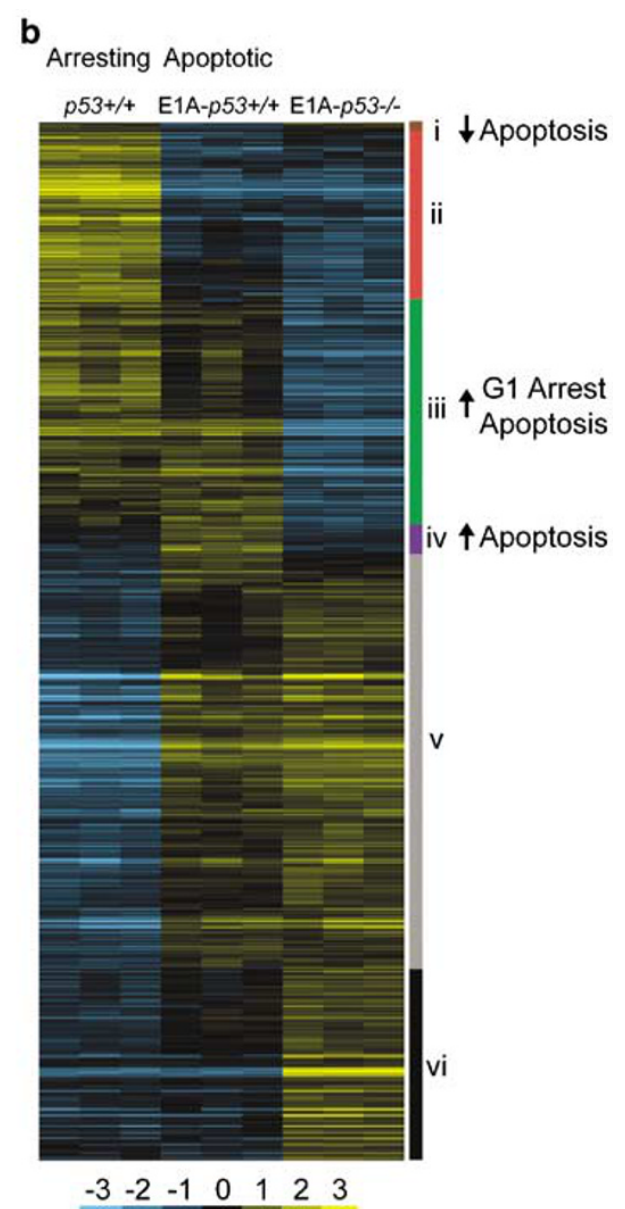

d Arresting Apoptotic

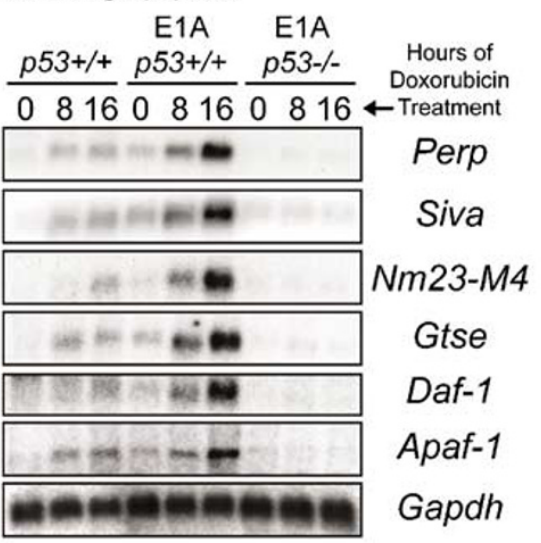


Table 1 Genes selectively induced during p53-dependent apoptosis using dChip analysis

\begin{tabular}{|c|c|c|c|c|}
\hline Accession & Gene name & Symbol & Apoptotic/E1A-p53-I- & Apoptotic/arresting \\
\hline Al854029 & p53 Apoptosis Effector Related to Pmp22 & Perp & 5.20 & 2.77 \\
\hline D63679 & Decay Accelerating Factor-1 & Daf-1 & 4.66 & 1.69 \\
\hline AF007267 & Phosphomannomutase 1 & Pmm1 & 3.62 & 1.47 \\
\hline L41365 & Decay Accelerating Factor-2 & Daf-2 & 2.82 & 1.41 \\
\hline AF110520 & H2-K Region Expressed gene & - & 3.01 & 1.94 \\
\hline AJ222580 & G2/S Phase Expressed Protein 1 & Gtse/B99 & 2.55 & 1.99 \\
\hline AW123567 & AW123567 & - & 2.34 & 1.46 \\
\hline Al836205 & Expressed in Non-Metastatic Cells 4 & Nm23-M4 & 2.43 & 2.35 \\
\hline D50264 & Phosphatidylinositol Glycan, class F & Pig-F & 2.57 & 1.66 \\
\hline AF085219 & Diacylglycerol Kinase, alpha & alpha-DGK & 2.44 & 1.99 \\
\hline Al834976 & Expressed sequence Al834976 & - & 2.02 & 1.98 \\
\hline M60348 & ATP-binding Cassette, subfamily B & Mdr & 2.36 & 1.53 \\
\hline AF06471 & Apoptotic Protease Activating Factor 1 & Apaf-1 & 2.30 & 1.38 \\
\hline AF033115 & CD27 Binding Protein & Siva & 2.04 & 1.48 \\
\hline AW012491 & AW012491 & - & 1.81 & 1.96 \\
\hline AW061272 & RIKEN cDNA 2410008J01 & - & 1.85 & 1.93 \\
\hline Al153421 & Al153421 & - & 2.09 & 2.15 \\
\hline AA985795 & RIKEN cDNA 2810407K09 & - & 1.74 & 1.67 \\
\hline AJ007909 & Erythroid Differentiation Regulator 1 & Edr & 2.24 & 2.16 \\
\hline X51905 & Lactate Dehydrogenase 2, $\mathrm{B}$ chain & Ldh-2 & 1.63 & 1.47 \\
\hline Al049206 & EST AI447490 (AW214473) & - & 1.56 & 1.50 \\
\hline AA869401 & Adenine Phosphoribosyl Transferase & Aprt & 1.70 & 1.52 \\
\hline U87240 & Mannosidase 2, alpha B1 & Man2b1 & 1.59 & 1.58 \\
\hline Al839817 & RIKEN cDNA 2810418105 & - & 1.56 & 1.37 \\
\hline L48514 & Paraoxonase 2 & Pon2 & 1.54 & 1.75 \\
\hline AW227620 & Proline Synthetase co-transcribed & - & 1.42 & 1.32 \\
\hline AA120675 & RIKEN cDNA 2610101M19 & - & 1.40 & 1.59 \\
\hline
\end{tabular}

Expression profiles of apoptotic E1A-p53+/+MEFs, arresting $p 53+/+$ MEFs, and E1A-p53-1- MEFs were generated for $\sim 12000$ murine genes and ESTs using the Affymetrix U74A Genechip. The experiment was performed at three different times using independently derived lines of MEFs, controlling for both experimental and biological variation. Data were analyzed using the DNA-Chip Analyzer (dChip) to identify genes upregulated selectively during apoptosis and in a p53-dependent manner. Value listed is fold-change. Genes were filtered according to several criteria, as described in the Materials and Methods. Those genes where the $90 \%$ confidence interval $(\mathrm{Cl})$ of the fold-increase is greater than 1.2 in both comparisons and whose mean expression difference was statistically significant by the unpaired $t$-test $(P<0.05)$ are shown, sorted by the lower boundary of the $\mathrm{Cl}$

Table 2 Genes selectively repressed during p53-dependent apoptosis using dChip analysis

\begin{tabular}{|c|c|c|c|c|}
\hline Accession & Gene name & Symbol & Apoptotic/E1A-p53-/- & Apoptotic/arresting \\
\hline $\begin{array}{l}\text { V00756 } \\
\text { AF017994 } \\
\text { AW060951 } \\
\text { Y08135 } \\
\text { X14309 }\end{array}$ & $\begin{array}{l}\text { Interferon-Related Developmental Regulator } 1 \\
\text { Mesoderm Specific Transcript } \\
\text { RIKEN cDNA } 1110001124 \text { gene } \\
\text { Acid Sphingomyelinase-like phosphodiesterase 3a } \\
\text { Solute Carrier Family } 3\end{array}$ & $\begin{array}{l}\text { Ifrd1 } \\
\text { Peg1/Mest } \\
\text { - } \\
\text { Asml3a } \\
\text { Slc3A2 }\end{array}$ & $\begin{array}{l}-2.18 \\
-2.18 \\
-1.83 \\
-1.94 \\
-1.33\end{array}$ & $\begin{array}{l}-1.76 \\
-2.91 \\
-1.70 \\
-1.55 \\
-1.33\end{array}$ \\
\hline
\end{tabular}

Expression profiles of apoptotic E1A-p53+/+ MEFs, arresting p53+/+ MEFs, and E1A-p53-/- MEFs were generated for $~ 12000$ murine genes and ESTs using the Affymetrix U74A Genechip. The experiment was performed at three different times using independently derived lines of MEFs, controlling for both experimental and biological variation. Data were analyzed using the DNA-Chip Analyzer (dChip) to identify genes downregulated specifically during apoptosis and in a p53-dependent manner. Value listed is fold-change. Genes were filtered according to several criteria, as described in the Materials and Methods. Those genes where the $90 \%$ confidence interval $(\mathrm{Cl})$ of the fold-decrease is greater than 1.2 in both comparisons and whose mean expression difference was statistically significant by the unpaired $t$-test $(P<0.05)$ are shown, sorted by the lower boundary of the $\mathrm{Cl}$

\footnotetext{
Figure 1 Microarray analysis to identify apoptosis-selective, p53-dependent genes. (a) Strategy for identifying apoptosis-specific p53 targets using MEFs. DNA damage $(0.2 \mu \mathrm{g} / \mathrm{ml}$ doxorubicin) treatment induces p53-mediated apoptosis in E1A-p53 + /+ MEFs, G1 arrest in p53 +/+ MEFs, or no p53 response in E1A-p53-/- MEFs. By comparing the expression profile of apoptotic E1A-MEFs to those of G1-arrested MEFs and of E1A-p53-deficient MEFs, p53-dependent genes selectively induced during apoptosis can be identified. (b) Expression profiles of 2248 genes and ESTs from apoptotic, G1-arrested, and E1A-p53-deficient MEFs were analyzed by hierarchical clustering, as described in the Materials and Methods. The complete experiment was performed three independent times, and the three columns for each genotype represent these individual replicates. For each gene, the degree of expression compared to the mean expression level for all samples is indicated by the scale below: yellow bars represent increased expression relative to the mean, whereas blue bars reflect decreased expression relative to the mean. Six distinct clusters ( $i-v i$, as indicated) were identified based on expression pattern. (c) Detailed view of p53 apoptotic cluster (cluster iv), with gene names indicated. Genes labeled in red were also identified by dChip analysis (Table 1). (d) Northern blot analysis of targets identified by microarray analysis using RNA from G1-arrested ( $p 53+/+$ ), apoptotic (E1A-p53+/+), and E1A-p53deficient MEFs, following 0,8 , or $16 \mathrm{~h}$ doxorubicin treatment. Perp (p53 apoptosis effector related to Pmp22), Siva (CD27 binding protein), Nm23-M4 (expressed in nonmetatstatic cells 4), Gtse (G two S phase expressed protein 1), Daf-1 (decay accelerating factor-1) and Apaf-1 (apoptotic protease activating factor-1) were examined. Gapdh serves as a loading control
} 
a

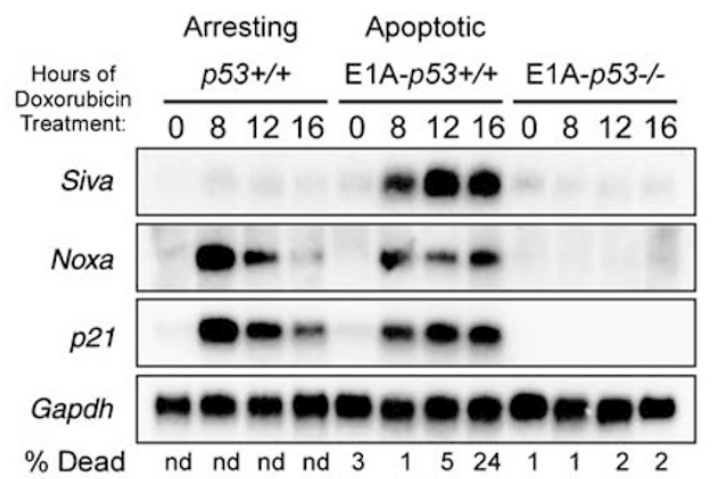

b
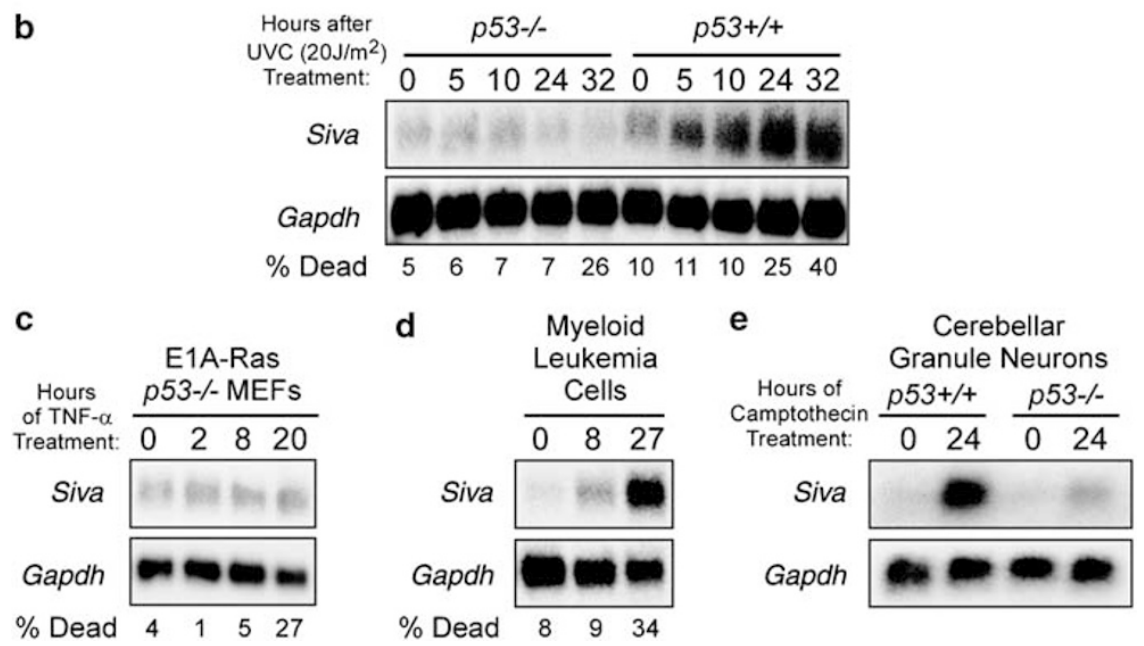

Figure 2 Siva is broadly induced during p53-dependent apoptosis. (a) Time course analysis of p53 target gene expression following doxorubicin treatment of MEFs correlates the upregulation of Siva, but not Noxa or p21, specifically with the induction of apoptosis in E1A-p53 + /+ MEFs. For all Northern blot analyses, Gapdh serves as a loading control. Where indicated below, the percentages of cell death were determined by trypan blue exclusion. (b) Siva expression is induced during UVC-activated, p53dependent apoptosis. Northern blot analysis shows RNA isolated from wild-type and p53-/- MEFs collected at different times following exposure to $20 \mathrm{~J} / \mathrm{m}^{2} \mathrm{UVC}$. (c) Siva expression is not induced during p53-independent apoptosis. Northern blot analysis shows RNA collected from E1A/Ras-expressing, p53-/- MEFs treated with TNF $\alpha$ for different periods of time. (d) Siva expression is induced in LTR6 myeloid leukemia cells undergoing p53-dependent apoptosis. LTR6 cells express a temperature-sensitive mutant of p53 (A135V) and undergo apoptosis upon activation of p53 at $32^{\circ} \mathrm{C}$. Northern blot analysis shows a time course of Siva induction following p53 activation in LTR6 cells. (e) Siva is induced in a p53-dependent manner in DNA damage-treated CGN. Northern blot analysis shows wild-type and p53-/- CGN treated with $10 \mu \mathrm{m}$ camptothecin for $24 \mathrm{~h}$

Siva is sufficient to reconstitute the p53 cell death response. The robust expression of Siva during p53dependent apoptosis, coupled with its reported capacity to induce cell death upon overexpression, ${ }^{19}$ suggested a role as a crucial mediator in the p53 apoptotic pathway. To define the contribution of Siva to the p53 cell death pathway, we tested the ability of Siva to substitute for p53 in apoptosis in E1A-p53-/- MEFs. We found that Siva induces apoptosis in E1A-p53-/- MEFs to levels commensurate with p53, $\sim$ 3-fold above background cell death observed with $\beta$ galactosidase expression (Figure 3). This finding demonstrates that expression of Siva is sufficient to recapitulate the p53 response, consistent with the idea that it participates in the $\mathrm{p} 53$ apoptotic program.

Siva is required for DNA damage-induced neuronal cell death. To examine the requirement for endogenous Siva in the p53 apoptotic program, we turned to the nervous system, a context in which Siva is induced (Figures 2e, 4e, and 6a and Fortin et al. $^{22}$ ) and where p53-dependent apoptosis has great physiological relevance in vivo. Various neuronal populations, including those in the cerebellum, cerebral cortex, and hippocampus, undergo p53-mediated apoptosis in response to diverse stress stimuli such as seizureassociated excitotoxicity, ischemia, and DNA damage. ${ }^{2}$ As Siva overexpression can induce apoptosis in CGN, ${ }^{22}$ we utilized these neurons to assess the requirement of Siva for p53-dependent cell death. Upon treatment with the DNAdamaging agent camptothecin, wild-type CGN undergo p53dependent apoptosis, a response that correlates with induction of both p53 and Siva (Figures 2e and 4a, b, e).

To establish the contribution of Siva to CGN apoptosis, we used RNA interference to knockdown Siva expression before induction of apoptosis. Among several short hairpin RNAs (shRNAs) generated, we identified one, Siva-B, which effectively reduces Siva expression (Figure 4c), and we utilized this in subsequent experiments. An shRNA directed against LacZ was used as a negative control (Supplementary Figure 4). We found that Siva protein levels are highly induced following camptothecin treatment in LacZ shRNA-expressing 

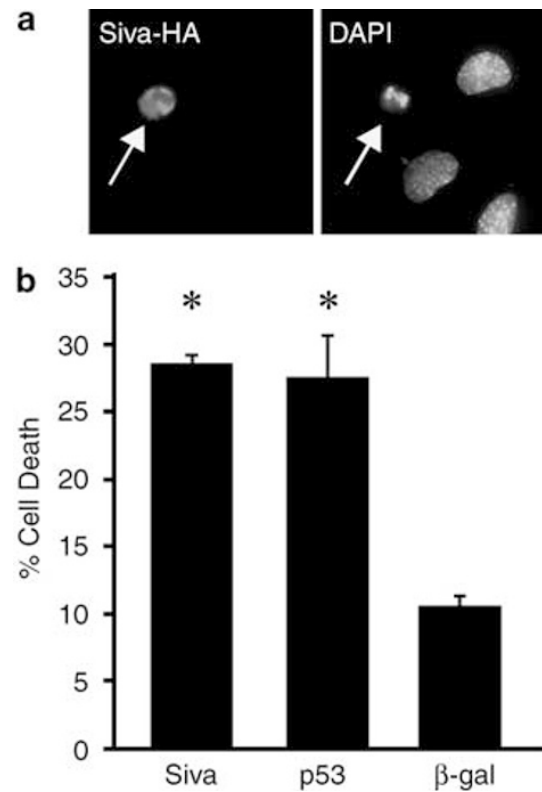

Figure 3 Siva expression is sufficient to induce apoptosis. (a, b) Siva-HA, p53, and $\beta$-galactosidase expression constructs were transiently transfected into E1A-p53-/- MEFs and apoptosis was examined $48 \mathrm{~h}$ later by assaying cells immunostained for each antigen (visualized using FITC-conjugated secondary antibodies) for the well-defined phenotypes of cell shrinkage and chromatin condensation, as assessed by DAPI staining. (a) Siva-HA-expressing cells (arrow) display apoptotic nuclear morphology by DAPI staining. (b) The percentages of Siva-HA, p53, and $\beta$-galactosidase expressing cells with apoptotic morphology are indicated. Data represent the average of five experiments, and at least 200 cells were counted in each experiment. Error bars represent standard error of the mean (S.E.M.). The bars marked with * are significantly different from the $\beta$-galactosidase control, as determined by one-way ANOVA $(P<0.001)$

CGN but not in Siva shRNA-expressing CGN, demonstrating the efficacy of knockdown (Figure 4e). Control neurons expressing the LacZ shRNA and treated with camptothecin display levels of apoptosis $\sim 5.6$-fold higher than DMSOtreated counterparts (Figure $4 \mathrm{f}$ and $\mathrm{g}$ ). In contrast, neurons expressing the Siva shRNA manifest an attenuated apoptotic response upon camptothecin treatment, with apoptosis levels only 2.4-fold higher than DMSO-treated neurons. These data demonstrate that reduced Siva expression confers resistance to DNA damage-induced neuronal cell death, in turn revealing that Siva is a pivotal component of the p53 neuronal apoptotic program.

Siva activates an apoptotic cascade paralleling that induced by DNA damage. To characterize the mechanism of Siva-mediated apoptosis, we examined features of apoptosis induced by Siva expression in comparison to cells undergoing p53-dependent apoptosis in response to DNA damage. In non-apoptotic cells, Bax resides in the cytosol, but following an apoptotic stimulus, translocates to the mitochondria where it oligomerizes, triggering mitochondrial membrane permeabilization. ${ }^{25}$ This relocalization is visualized by the appearance of puncta corresponding to mitochondria. Disruption of the mitochondrial membrane results in cytochrome $c$ release ${ }^{25}$ manifested as diffuse cytosolic staining and loss of mitochondrial staining. Finally, a more terminal step in the apoptotic process is the cleavage and activation of Caspase- $3{ }^{25}$ To identify the point at which Siva acts to stimulate apoptosis, we introduced Siva-HA into both E1A-p53-/- MEFs and CGN and co-stained cells for Siva and Bax, cytochrome $c$, or cleaved Caspase-3. Mirroring the endogenous p53-apoptotic cascade in DNA damage-treated E1A-p53+/+ MEFs and CGN (Figure 5a, Supplementary Figure 5a), Siva-expressing apoptotic cells exhibit Bax translocation to the mitochondria, cytochrome $c$ release, and Caspase-3 activation (Figure 5b, Supplementary Figure 5b). To establish genetically that Siva acts upstream of mitochondrial permeabilization, we examined the ability of Siva to induce apoptosis in E1A-expressing Bax-/-;Bak-/- MEFs. As with p53, Siva is incapable of inducing apoptosis in Bax-, Bakdouble-mutant cells (Figure 5c), providing genetic evidence that mitochondrial membrane disruption through Bax and Bak is required for both Siva- and p53-mediated apoptosis. Our data indicate that the pathway of Siva-induced apoptosis mimics the endogenous p53-apoptotic response and demonstrate that Siva is poised upstream of events at the mitochondria.

Signaling from the plasma membrane is important for neuronal apoptosis. Previous experiments examining overexpressed, epitope tagged-Siva have produced conflicting data regarding its localization, showing either diffuse cytoplasmic localization with some mitochondrial targeting, or, alternatively, nuclear localization. ${ }^{26,27}$ These data are in contrast to the initial characterization of Siva as a TNF receptor-interacting protein, which suggested plasma membrane localization. ${ }^{19}$ To clarify Siva's mode of action, we sought to determine where endogenous Siva is localized during apoptosis. Because of Siva's functional importance for apoptosis in neurons, we performed subcellular fractionation on irradiated brain, where p53dependent apoptosis occurs in vivo following treatment with ionizing radiation, ${ }^{28}$ and where we observed robust Siva protein induction upon $\gamma$-irradiation (Figure 6a). Using differential centrifugation, Siva fractionated in a pattern closely resembling that of the plasma membrane, indicated by epidermal growth factor receptor (EGFR) (Figure 6b). We obtained further support for Siva's membrane localization by fractionating proteins by flotation density in an iodixanol gradient (Figure 6c). Siva showed a distribution profile indistinguishable from the $\mathrm{Na}+\mathrm{K}+$ ATPase plasma membrane marker and distinct from the Hsp60 mitochondrial marker. Together, these data provide the first clear evidence that endogenous Siva is associated with the plasma membrane during apoptosis.

The two major apoptotic pathways are the intrinsic mitochondrial pathway and the extrinsic death-receptor pathway. The plasma membrane localization of Siva, coupled with the initial identification of Siva as a TNF receptor-interacting protein, suggested that Siva could act via the extrinsic pathway. The requirement for Siva in the p53 apoptotic response of CGN to DNA damage suggested further the importance of membrane signaling events in p53-dependent apoptosis, which is generally considered to occur through the intrinsic pathway. Engagement of the extrinsic pathway relies initially on death receptor-induced Caspase-8 cleavage and activation. Caspase-8, in turn, promotes apoptosis either 


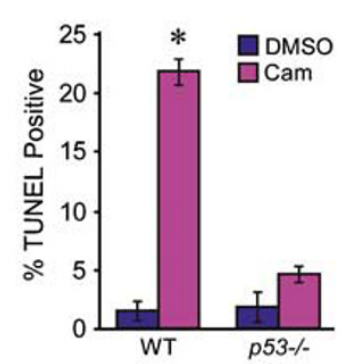

C

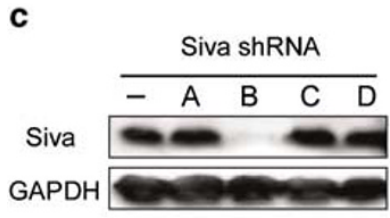

WT

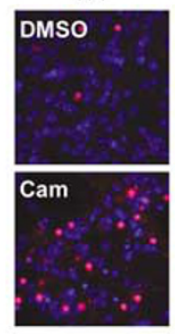

d LacZ shRNA

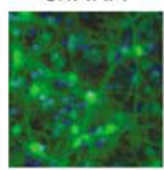

b
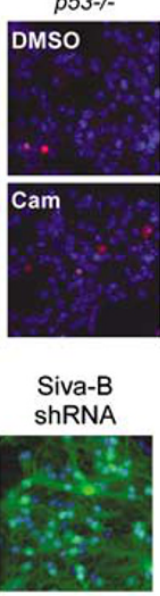

e

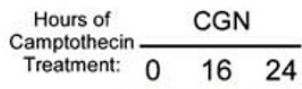

p53

Tubulin
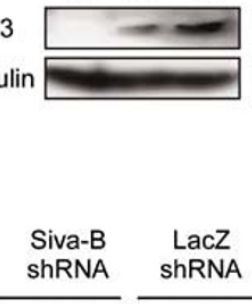

Tubulin
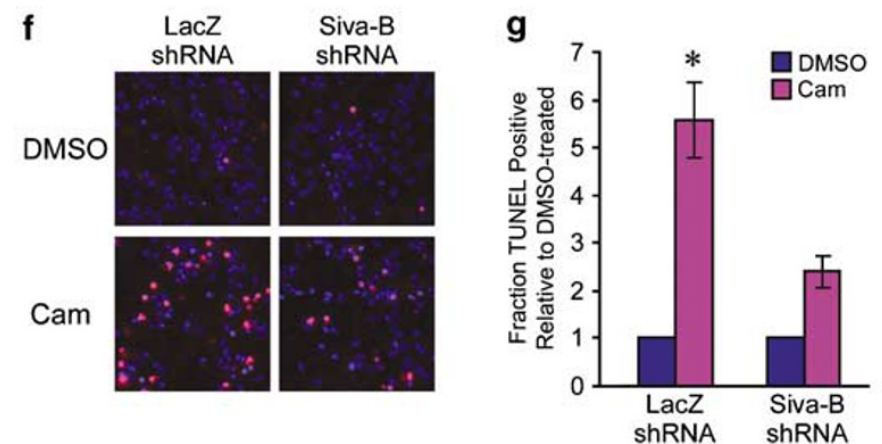

Figure 4 Siva is required for the p53-dependent apoptotic response of CGN to DNA damage. (a) CGN treated with camptothecin undergo p53-dependent apoptosis. Wildtype and $p 53-/-$ CGN cultured for 3 DIV were treated with either DMSO as a control or $10 \mu \mathrm{M}$ camptothecin for $24 \mathrm{~h}$. Immunofluorescent TUNEL staining was used to quantify apoptosis (red) amongst all nuclei (DAPI, blue). Graphed data represent the average of three individual experiments. Error bars indicate S.E.M. The * indicates that the percentage of apoptosis in the camptothecin-treated WT CGN is significantly different from all other samples, as determined by one-way ANOVA $(P<0.001)$. (b) Western blot analysis of CGN cultured for 3 DIV and then treated with DMSO for $24 \mathrm{~h}$ or $10 \mu \mathrm{M}$ camptothecin for the indicated times demonstrates DNA damage-induced activation of p53 for $24 \mathrm{~h}$. $\alpha$-Tubulin serves as a loading control. (c) The Siva-B shRNA effectively attenuates Siva expression. Western blot analysis of protein extracts from H1299 cells cotransfected with plasmids expressing HA-Siva and the indicated Siva shRNAs. Siva was detected using an HA antibody. Gapdh serves as a loading control. (d) GFP expression demonstrates the efficiency of infection of CGN by lentiviruses expressing LacZ and Siva-B shRNAs. The lentiviral vector used to express the shRNAs also expresses CMV-driven GFP, thereby allowing assessment of infection efficiency. Based on GFP expression, we estimate that approximately $90 \%$ of the CGN were infected in each experiment. (e) Western blot analysis of CGN following lentivirus-mediated expression of LacZ or Siva-B shRNAs demonstrates knockdown of endogenous Siva protein. Wild-type CGN were transduced at 2 DIV with Siva or LacZ shRNA-expressing lentiviruses and treated at 6 DIV with DMSO or camptothecin for $24 \mathrm{~h}$. $\alpha$-Tubulin serves as a loading control. (f) Representative images of TUNEL staining of DMSO- or camptothecin-treated CGN following infection with lentiviruses expressing LacZ or Siva-B shRNAs. Apoptotic cells (red) are visible amongst non-apoptotic nuclei (DAPI, blue). (g) Quantification of neuronal apoptosis shown in (f). The percentage of TUNEL-positive neurons in camptothecin-treated CGN is shown relative to the percentage of TUNEL-positive neurons in control DMSO-treated CGN for each lentiviral infection condition. The level of death observed in the LacZ shRNA-expressing neurons is comparable to that seen in uninfected and empty-lentivirus infected neurons treated with camptothecin (data not shown). At least 1000 cells were counted for each condition per experiment. Data represent the average \pm S.E.M. of five independent experiments. The sample marked with * is significantly different from all others, as determined by one-way ANOVA $(P<0.001)$

through direct activation of effector caspases, such as Caspase-3, or through cleavage of Bid to truncated Bid (tBid), which transits to the mitochondria to stimulate the intrinsic apoptotic pathway, via effects on Bax and Bak. To determine if death-receptor signaling is indeed involved in p53-dependent neuronal apoptosis, we first examined whether hallmarks of extrinsic pathway activation, including cleaved Caspase-8 and tBid, were observed. Western blot analysis of camptothecin-treated CGN revealed the presence of cleaved, active Caspase-8 (p18) as well as tBid (Figure 6d), demonstrating activation of death-receptor signaling during this p53-dependent apoptotic program. To determine the role of the extrinsic pathway in p53-induced apoptosis, we examined the requirement for Caspase-8 and Bid in camptothecin-stimulated cell death in CGN. Campthothecin-treated CGN were concurrently treated with a Caspase-8 inhibitor (Z-IETD-fmk), or with broad spectrum caspase inhibitors (Boc-D-fmk and Z-VAD$\mathrm{fmk}$ ). We found that apoptosis is nearly completely blocked in neurons treated with the Caspase- 8 inhibitor, to an extent similar to that achieved using the broad spectrum caspase inhibitors (Figure 6e), suggesting that Caspase-8 is important for p53-dependent neuronal apoptosis in response to DNA damage. We assessed the importance of Bid in this pathway by examining apoptosis in camptothecin-treated Bid-deficient CGN. Cell death in Bid-/- CGN was attenuated compared to WT CGN (Figure 6f). Although Bid has been shown to be important for apoptosis in the adult brain following ischemic injury, ${ }^{29}$ these results reveal a novel role for Bid in DNA 
a

DNA damage-induced, p53-dependent apoptosis
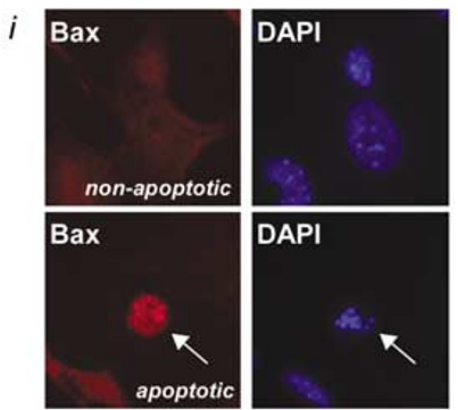

ii

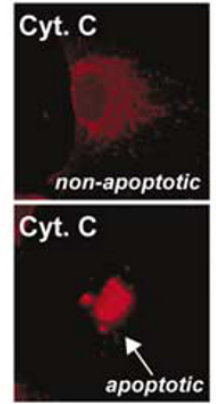

iii

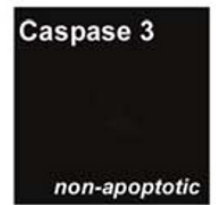

Caspase 3

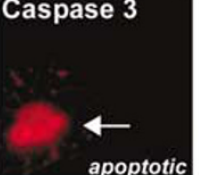

apoptotic
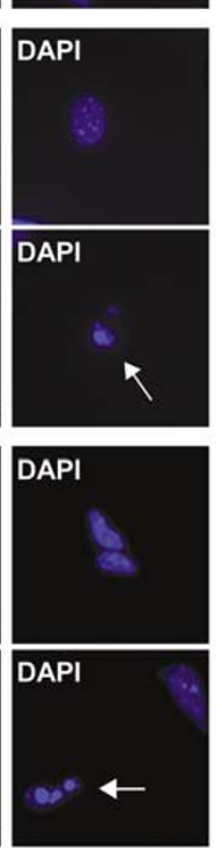

b

Siva-induced apoptosis
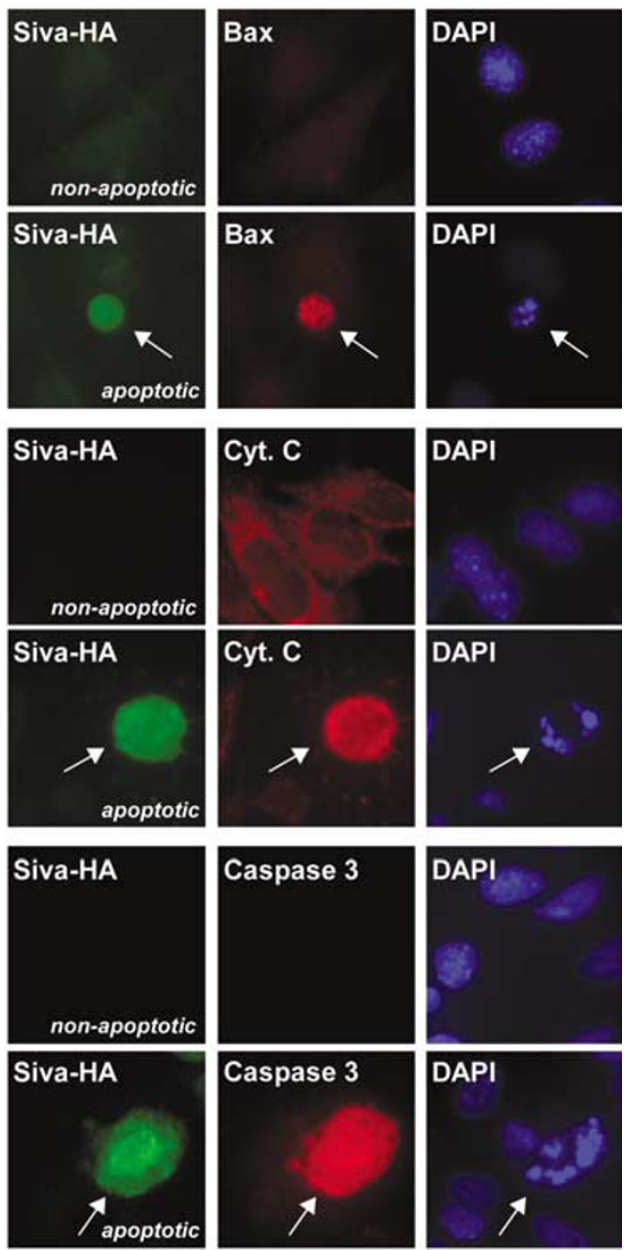

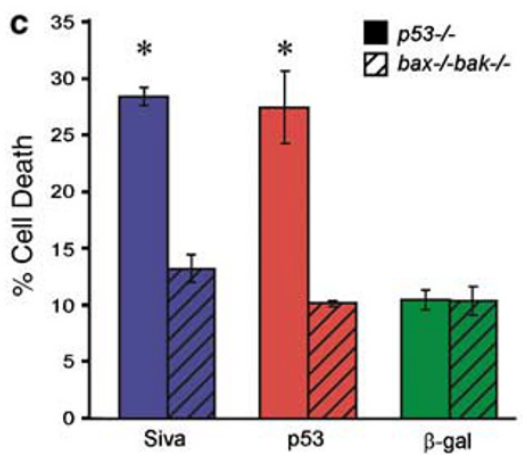

Figure 5 Siva activates an apoptotic cascade paralleling that induced by p53 in response to DNA damage. (a, $\mathbf{b})$ Siva induces features of apoptosis mirroring DNA damage-triggered apoptosis in E1A-p53+/+ MEFs. E1A-p53+/+ MEFs treated with $0.2 \mu \mathrm{g} / \mathrm{ml}$ doxorubicin for $16 \mathrm{~h}$ to induce apoptosis (a) and E1A-p53-/- MEFs transfected with Siva-HA for $48 \mathrm{~h}$ (b) were immunostained for Bax (I), cytochrome $c$ (ii), and cleaved Caspase-3 (iii) to examine features of apoptosis. DAPI staining shows chromatin condensation. Apoptotic and non-apoptotic cells are indicated. (c) Siva-induced apoptosis requires Bax and Bak. Siva-HA, p53, and $\beta$-galactosidase constructs were transiently transfected into E1A-p53-/- (solid bars) and E1A-Bax-/-;Bak-/- (hatched bars) MEFs, and after $48 \mathrm{~h}$ cells staining positively for each antigen by immunofluorescence were assayed for apoptosis by chromatin condensation, as assessed by DAPI staining. The percentages of Siva-HA-, p53-, and $\beta$-galactosidaseexpressing cells with apoptotic morphology are graphed. Data represent the average of at least four experiments, and at least 200 cells were counted in each experiment. Error bars represent S.E.M. The values marked with * are significantly different from all other values, as determined by one-way ANOVA $(P<0.001)$

damage-induced, p53-dependent neuronal cell death. Our findings suggest that in neurons, the p53 apoptotic cascade triggered by DNA damage involves membrane signaling events, mediated via Siva and via the Caspase-8/Bid axis, underscoring the importance of the extrinsic pathway in this context of p53-dependent cell death. 
a

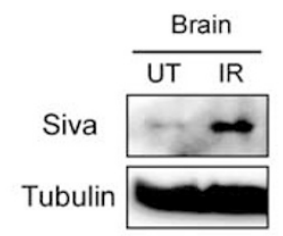

b b $\quad$\begin{tabular}{c}
\multicolumn{4}{c}{$\gamma$-IR treated brain } \\
\cline { 2 - 4 }
\end{tabular}

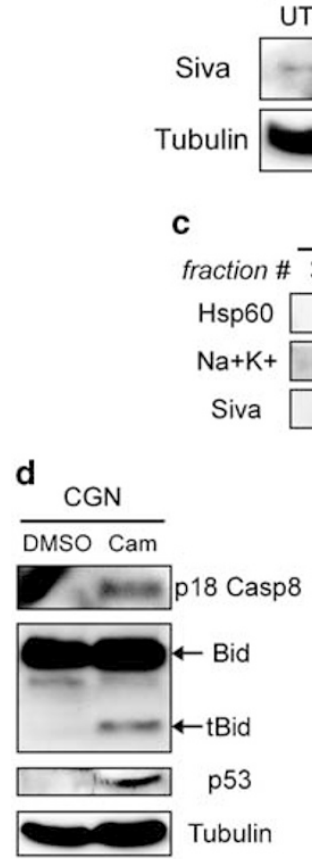

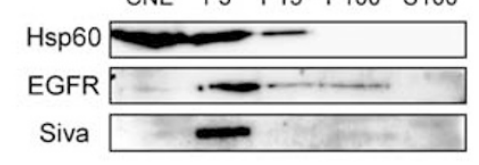

$\gamma$-IR treated brain

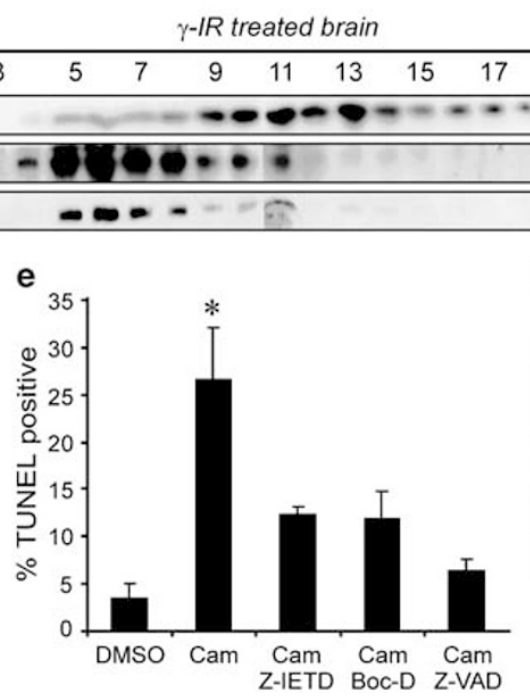

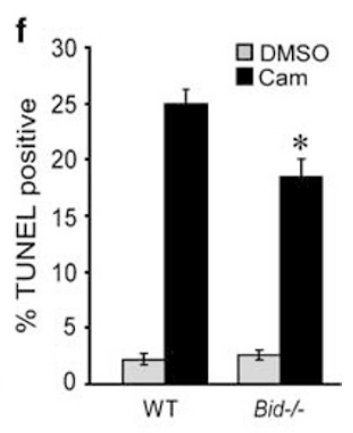

Figure 6 p53-dependent neuronal cell death involves Siva localization to the plasma membrane, as well as Caspase-8 and Bid. (a) Siva protein expression in the brain is induced during p53-dependent cell death in vivo. Western blot analysis demonstrates Siva induction in whole brain tissue $16 \mathrm{~h}$ following treatment with $10 \mathrm{~Gy}$ ionizing radiation. $\alpha$-Tubulin serves as a loading control. (b) Subcellular fractionation by differential centrifugation of brain tissue $16 \mathrm{~h}$ following 14 Gy ionizing radiation. Brain extracts were separated into CNE, P3 (heavy membrane), P15 (light membrane), P100 (microsomal), and S100 (cytosolic) fractions. Western blot analysis for Siva, EGFR (plasma membrane marker), and Hsp60 (mitochondrial marker) demonstrates plasma membrane localization of endogenous Siva. (c) Fractionation of irradiated brain tissue (10 Gy, $17 \mathrm{~h}$ post-treatment) by iodixanol (Optiprep) gradient shows that Siva co-distributes with plasma membrane, indicated by the $\mathrm{Na}+\mathrm{K}+\mathrm{ATPase}$ plasma membrane marker, rather than with mitochondria, indicated by Hsp60. (d) The extrinsic pathway is activated in camptothecin-treated CGN. Western blot analysis comparing wild-type CGN treated for $48 \mathrm{~h}$ with camptothecin and undergoing apoptosis to non-apoptotic, DMSO-treated CGN. The generation of cleaved, active Caspase-8 and tBid is observed in camptothecin-treated CGN. p53 induction by camptothecin is also shown. $\alpha$-Tubulin serves as a loading control. (e) Caspase- 8 is required for the apoptotic response of CGN to DNA damage. CGN were treated for $24 \mathrm{~h}$ with $10 \mu \mathrm{m}$ camptothecin in the absence or presence of $20 \mu \mathrm{m}$ Caspase-8 (Z-IETD-fmk) or pan-caspase (Boc-D-fmk and Z-VAD$\mathrm{fmk}$ ) inhibitors, and apoptosis was assayed by TUNEL staining. At least 1000 cells were counted per treatment per experiment. Data represent the average \pm S.E.M. of three independent experiments. The value marked with * is significantly different from all other values, as determined by one-way ANOVA $(P<0.05)$. (f) Bid is an important component of the DNA damage-induced, p53-dependent apoptotic response in neurons. Wild-type and Bid-/ - CGN cultured for 3 DIV were treated with either DMSO as a control or $10 \mu \mathrm{M}$ camptothecin for $24 \mathrm{~h}$. Immunofluorescent TUNEL staining was used to quantify apoptosis among all nuclei. Data represent the average of three individual experiments. Error bars indicate S.E.M. The percentage of cell death in the camptothecin-treated Bid-/- CGN, marked by the *, is significantly different from the percentage of cell death in camptothecin-treated WT CGN, as determined by one-way ANOVA $(P<0.01)$

\section{Discussion}

Despite the central role of p53-mediated apoptosis in both tumor suppression and the response to neuronal injury, many facets of p53-dependent cell death remain incompletely understood. Here, to better understand the pathway leading to apoptosis, we used gene expression profiling to identify genes selectively activated by p53 during apoptosis compared to $\mathrm{G} 1$ arrest. Strikingly, the number of p53 target genes with enriched expression during apoptosis in MEFs is very limited, representing only $14 \%$ of p53-dependent genes, indicating that there are few genes that may be involved in directing the apoptotic cell fate. These apoptosis-associated genes are largely novel, encoding proteins with a variety of cellular functions, and do not include canonical p53 targets such as Bax. This portrait of global gene expression patterns associated with p53-induced apoptosis lays the foundation for further elaboration of the p53 apoptotic program in the future.
We focused on one particular apoptosis-specific gene identified by our array analysis, Siva, which was notable because it encodes a proapoptotic protein unrelated to known apoptosis effectors, and which therefore could provide novel insight into p53's mode of action. Consistent with a role as a p53 effector, Siva expression is highly induced during p53dependent, but not p53-independent, apoptosis in various contexts, and Siva triggers an apoptotic cascade similar to p53. To assess the requirement of Siva for apoptosis, we turned to a physiological system of DNA damage-induced neuronal apoptosis, in which p53 apoptotic pathway components at or downstream of mitochondria are relatively wellestablished, but where those components more proximal to p53 that initiate apoptosis are not well-defined. ${ }^{4}$ Using this model, we provided the first evidence that Siva is required for p53-dependent apoptosis and showed further that Siva acts upstream of mitochondrial dysfunction. Although Siva is important for neuronal cell death, apoptosis in the absence 
of Siva is only partially compromised, indicating that it collaborates with other effectors. These could be proapoptotic p53 target genes already implicated in neuronal apoptosis, including Apaf-1, Perp, Puma, and Bax, ${ }^{10,11,14,30,31}$ or potentially novel target genes. Beyond its pivotal role in apoptosis in DNA damage-treated neurons, the broad p53dependent activation of Siva during apoptosis in diverse cell types indicates that Siva may serve as a mediator of p53induced cell death in multiple settings.

Although Siva has been established previously to induce apoptosis when overexpressed, ${ }^{19,22,26}$ the mechanism by which Siva engenders cell death has been controversial. Siva was originally identified as an interacting protein for certain TNF-receptors, including CD27 and GITR. ${ }^{19,32}$ However, subsequent studies suggested that Siva functions at the mitochondria via interaction with antiapoptotic $\mathrm{Bcl}-2$ family members. ${ }^{27,33}$ In contrast, a report examining Siva-induced apoptosis in T-lymphocytes found exogenously introduced GFP-tagged Siva localized to unidentified nuclear substructures. ${ }^{26}$ Given these disparate findings, clarification of endogenous Siva localization during apoptosis was crucial for understanding how Siva triggers cell death. Our demonstration that endogenous Siva associates with the plasma membrane during DNA damage-induced, p53-initiated neuronal cell death suggests that this p53 apoptotic program involves expression of Siva, which, likely through association with TNF-receptors, participates in extrinsic pathway signaling. The extrinsic pathway is further implicated in p53 neuronal apoptosis by our findings that Caspase- 8 and Bid both contribute to this cell death response. Although p53mediated apoptosis has been considered to occur primarily through activation of the intrinsic pathway, our work supports growing evidence that p53 also engages the extrinsic pathway. Specifically, p53 directly stimulates expression of several components of the death-receptor pathway, including the death-receptors Fas and Killer, the adaptor protein TRAF4, and Bid, and genetic evidence supports the requirement for some of these in p53-dependent apoptosis. ${ }^{34-36}$

In the extrinsic pathway, engagement of death receptors leads to apoptosis via direct activation of caspase-3, or alternatively, through signaling between the plasma membrane and mitochondria to engage the intrinsic pathway. Our observation that Siva, a mediator of cell death acting at the membrane, requires Bax/Bak to induce apoptosis suggests the significance of communication between the membrane and the mitochondria for p53-dependent apoptosis. Moreover, the observed requirement for Bid highlights the importance of crosstalk between the extrinsic and intrinsic pathways for neuronal cell death. However, we observe that DNA damage-induced neuronal apoptosis is only partially compromised in the backdrop of Bid-deficiency, reminiscent of the partially impaired cell death observed in Bid null thymocytes and MEFs exposed to FasL or TNF $\alpha$, respectively. ${ }^{37}$ These findings implicate collateral signals, either emanating from the membrane or acting directly via the intrinsic pathway, for the complete execution of an apoptotic response. Siva's specific role with respect to Caspase-8 and Bid remains to be determined, but it may act in a parallel pathway, sending an additional signal from the membrane to the mitochondria to promote apoptosis. Alternatively, it may act in a linear pathway, serving to promote Caspase-8 activity and Bid action. Future experimentation will distinguish these possibilities.

Although the precise genetic and environmental factors that initiate p53 activation in different neurological disorders vary, the outcome of neuronal death is a shared consequence and results in many of the symptoms manifested in these diseases, such as cognitive impairment and motor dysfunction. $^{38}$ While studies in neuronal disease models have indicated that the apoptotic cascade leads to compromised mitochondrial function, the specific effectors upstream of mitochondria have not been clearly established. Siva is an upstream regulator of mitochondrial dysfunction whose expression in the brain is induced not only by DNA damage but also by focal ischemia in a stroke model, ${ }^{22}$ supporting the notion that Siva may play an important role in mediating p53-dependent brain damage following acute injury. Future investigation will define the contexts in which Siva is involved in the pathogenesis of neuronal injury and neurodegenerative diseases. As a critical signaling component in neuronal apoptosis, Siva represents a potential therapeutic target for the treatment of neuronal cell death diseases.

\section{Materials and Methods \\ Preparation of apoptotic and G1-arrested populations and hybridization to oligonucleotide arrays. The generation of MEFs and preparation of apoptotic, G1-arrested, and p53-deficient populations have been described previously. ${ }^{15}$ In brief, to generate the apoptotic population, wild-type $M E F s$ were infected with retroviruses carrying the E1A oncogene (E1A-MEFs) and then treated with $0.2 \mu \mathrm{g} / \mathrm{ml}$ doxorubicin (Sigma) for $16 \mathrm{~h}$ to induce apoptosis. E1A- expressing p53-/ $\mathrm{MEFs,} \mathrm{which} \mathrm{fail} \mathrm{to} \mathrm{undergo} \mathrm{apoptosis,} \mathrm{were} \mathrm{treated} \mathrm{similarly} \mathrm{to}$ control for E1A expression and to identify p53-dependent genes. To obtain the G1-arrested population, wild-type MEFs were synchronized by serum starvation, released, and treated with $0.2 \mu \mathrm{g} / \mathrm{ml}$ doxorubicin for $12 \mathrm{~h}$. RNA was isolated from MEFs using RNeasy columns (Qiagen) and processed according to the Affymetrix, protocol. Hybridization to the murine U74A and U74B GeneChip oligonucleotide arrays, encompassing $\sim 24000$ genes and ESTs (Affymetrix), was performed by the Stanford University PAN facility.}

Cluster analysis. We combined the U74A and U74B data sets and performed the analysis using the software program pcl-analysis. The data were converted into log-ratio form by dividing each measurement by the mean for that gene across all experiments and then taking the $\log _{2}$. The data were then centered to show deviations from the mean by calculating the mean log ratio across the $p 53+/+$, $\mathrm{E} 1 \mathrm{~A}-\mathrm{p} 53+/+$, and $\mathrm{E} 1 \mathrm{~A}-\mathrm{p} 53-/-$ experiments and subtracting this mean from each of the three expression values. This was performed independently for each of the three replicate experiment sets. To identify and remove genes with high background variability, we generated a noise score by calculating the ratio of the variance between conditions to the mean variance across the replicates of each condition (between/within variance). ${ }^{39}$ Genes with high between/within variance scores are more likely to show true expression differences relative to noise. To quantify this, we repeated the analysis with a data set that had been randomly shuffled within each row to give a conservative estimate of the false-positive rate. Based on this analysis, we found that selecting genes based on a between/within variance cutoff of 3 gave 2248 genes with an estimated false-positive rate of $4.8 \%$. Thus, over $95 \%$ of these genes are predicted to have significantly greater expression differences between the conditions tested than can be explained by random noise.

dChip analysis. The CEL files generated by the Affymetrix Microarray Suite version 4.0 were converted into DCP files using dChip. The DCP files were normalized and gene expression data analyzed using dChip. Apoptotic samples were designated 'experimental' $(E)$ and independently compared to both the G1-arrested samples and the E1A-p53-/- samples, designated as 'baseline' (B). Genes whose expression was higher in apoptosis when compared to both the 
G1-arrested and E1A-p53-/- populations were identified using the following filtering criteria: $E / B>1.2$, using the lower boundary of the $90 \%$ confidence interval of the fold-change; $E-B>100$; and $P(E-B) \leqslant 0.05$.

Northern blot analysis. RNA was prepared using Trizol reagent (Invitrogen) and Northern blotting performed using standard methods. cDNAs for murine Siva, Perp, Nm23-M4, Gtse, Daf-1, Apaf-1, Noxa, p21 and Gapdh served as probes.

Cell death assay in MEFs. The Siva-HA expression vector was generated by PCR amplifying the Siva ORF from a Siva-containing I.M.A.G.E. clone using primers containing Sall sites and cloning into the KA mammalian expression vector. ${ }^{15}$ The p53 and $\beta$-galactosidase expression vectors have been described ${ }^{40} \mathrm{E} 1 \mathrm{~A}$ expressing MEFs were plated and transfected as described previously. ${ }^{15}$ Siva$\mathrm{HA}, \mathrm{p} 53$, and $\beta$-galactosidase-expressing cells were detected using antibodies against HA (12CA5 or rabbit anti-HA, Zymed, 1:100), p53 (DO-1, Santa Cruz, $1: 500)$, and $\beta$-galactosidase $\left(5^{\prime}-3^{\prime}, 1: 50\right)$, respectively, and the percentages of apoptotic nuclei were determined by DAPI staining and examining for chromatin condensation, which correlates with DNA fragmentation as assessed by TUNEL. ${ }^{40}$ Antibodies against Bax (Santa Cruz, 1:50), cytochrome $c$ (Zymed, 1:50), and cleaved Caspase-3 (Cell Signaling, 1:100) were also used to examine apoptosis.

Primary neuronal cultures, lentivirus infection, and RNA interference. CGN were prepared from mice on post-natal days 5-7 (P5$\mathrm{P} 7)$, as described. ${ }^{41} \mathrm{CGN}$ were treated with $10 \mu \mathrm{M}$ camptothecin or DMSO control at 3 days in vitro (DIV) and collected at various times following treatment. Protein samples were collected in lysis buffer containing $1 \%$ SDS, $100 \mathrm{mM} \mathrm{NaCl}$, and $10 \mathrm{mM}$ Tris pH 7.6. For caspase inhibitor studies, $20 \mu \mathrm{M}$ inhibitor or DMSO control was added to the media at the same time as camptothecin treatment. Caspase inhibitors (Calbiochem) used were Z-IETD-fmk (Caspase-8) and Boc-D-fmk and ZVAD-fmk (broad spectrum). Apoptosis was detected by the TUNEL assay. ${ }^{40}$

For RNA interference experiments, oligonucleotides encoding shRNAs directed against Siva and LacZ were cloned into the LL3.7 lentiviral vector as described. ${ }^{42}$ The Siva-B hairpin sequence is $5^{\prime}$-GATTGTTCCGTGAACCACC-3'. The LacZ shRNA sequence is $5^{\prime}$-TCGCTGATTTGTGTAGTCG- $3^{\prime}$. To generate lentiviruses, 293T cells were transfected with Siva-B-LL3.7 or LacZ-LL3.7 along with viral packaging vectors, using the calcium phosphate method. Wild-type CGN were infected with viral supernatant at 2 DIV for $8 \mathrm{~h}$ and then returned to conditioned media. Four days after introduction of the shRNAs (6 DIV), to allow adequate time for the shRNAs to be produced and knockdown to occur, CGN were treated with DMSO or $10 \mu \mathrm{m}$ camptothecin for $24 \mathrm{~h}$. Neurons were collected for Western blotting in lysis buffer containing $20 \mathrm{mM}$ Tris $\mathrm{pH} 8,150 \mathrm{mM} \mathrm{NaCl}, 1 \mathrm{mM}$ EDTA, $1 \%$ Triton $\mathrm{X}-100,0.5 \% \mathrm{NP}-40$, and $9 \mathrm{M}$ urea or fixed for TUNEL staining.

Western blot analysis. Whole brain extracts were prepared from P6-P7 mice either left untreated or treated with ionizing radiation. Brain tissue was extracted in lysis buffer containing $9 \mathrm{M}$ urea, and protein concentrations were determined using the BCA Protein Assay Kit (Pierce). Western blotting was performed using standard techniques, with antibodies against p53 (CM5, Novacastra, 1:2000), Bid (courtesy of D Huang, $1: 250$ ), Caspase-8 (courtesy of M Bogyo, $1: 1000$ ), GAPDH (RDI, $1: 5000$ ), and $\alpha$-tubulin (Sigma, 1:10000). The antibody directed against murine Siva was raised in rabbits against a peptide with the sequence ELSHGVFAERYSREVFER (Zymed) and affinity purified using the SulfoLink Kit (Pierce).

Subcellular fractionation. For differential centrifugation, whole brains were dissected from P6 mice $16 \mathrm{~h}$ following treatment with $14 \mathrm{~Gy}$ ionizing radiation. Brains were homogenized using a dounce homogenizer in a buffer containing $10 \mathrm{mM}$ HEPES pH 7.4, 2 mM EDTA, 0.32 M sucrose, and protease inhibitor cocktail (Roche). Cell homogenates were centrifuged sequentially at $600 \times g$ for $10 \mathrm{~min}$ to remove nuclei and unbroken cells, $3000 \times g$ for $10 \mathrm{~min}$ to pellet plasma membrane sheets and heavy mitochondria (P3), $15000 \times g$ for 15 min to yield the light mitochondria-enriched fraction (P15), and $100000 \times g$ for $1 \mathrm{~h}$ to yield the microsomal (P100, pellet) and cytosolic ( $\mathrm{S} 100$, supernatant) fractions. At each speed, the supernatants were centrifuged twice and the pellets washed by resuspension in homogenization buffer and recentrifugation. P3, P15, and P100 pellets were lysed in a buffer containing $1 \%$ NP- $40,1 \%$ Triton X-100, $0.5 \%$ SDS, $0.5 \%$ sodium deoxycholate, $5 \mathrm{mM}$ EDTA, $5 \mathrm{mM}$ EGTA, and protease inhibitors. Crude nuclear extracts (CNE) were extracted from the $600 \times g$ pellet in a high-salt nuclear extraction buffer consisting of $20 \mathrm{mM} \mathrm{HEPES} \mathrm{pH} \mathrm{7.9,2} \mathrm{mM} \mathrm{MgCl} 2,10 \mathrm{mM}$
$\mathrm{KCl}, 420 \mathrm{mM} \mathrm{NaCl}, 0.1 \% \mathrm{NP}-40,0.1 \mathrm{mM}$ EDTA, $0.1 \mathrm{mM}$ EGTA, 20\% glycerol, and protease inhibitor cocktail. For gradient experiments, brains from 3-week-old irradiated mice (10 Gy, 17 h) were fractionated using a self-forming linear 10-20$30 \%$ iodixanol gradient, as described. ${ }^{43}$ Equal volumes of each fraction were analyzed by Western blotting. We used antibodies directed against Siva, EGFR (courtesy of S Pfeffer, $1: 1000$ ), NA +K + ATPase (courtesy of WJ Nelson, 1:1000), and Hsp60 (StressGen, 1:4000).

Acknowledgements. We are grateful to Anne Brunet, Julien Sage, Tom Johnson, Rebecca Ihrie, Steven Artandi, and Amato Giaccia for helpful comments and critical reading of the paper. We thank Jim Brown for assistance with dChip analysis, Roger Vogelmann for assistance with the iodixanol gradients, and Anne Brunet for help with neuronal preparations and lentiviral infections. The Bax-/Bak-/- MEFs and Bid-/- mice were kindly provided by Craig Thompson and Stanley Korsmeyer, respectively. The EGFR, $\mathrm{Na}+\mathrm{K}+$ ATPase, Bid, and Caspase-8 antibodies were provided by Suzanne Pfeffer, W James Nelson, David Huang, and Matthew Bogyo, respectively. This work was supported by the $\mathrm{NCl}$ (PHS Grant Number CA09302-27) and Giannini Family Foundation to SBRJ, the Susan G Komen Foundation to SB, a Howard Hughes Medical Institute pre-doctoral fellowship to JIM, and the Damon Runyon Cancer Research Foundation and the Donald E and Delia B Baxter Foundation to LDA.

1. Vousden KH, Lu X. Live or let die: the cell's response to p53. Nat Rev Cancer 2002; 2 594-604.

2. Morrison RS, Kinoshita Y, Johnson MD, Guo W, Garden GA. p53-dependent cell death signaling in neurons. Neurochem Res 2003; 28: 15-27.

3. Bae $\mathrm{Bl}, \mathrm{Xu} \mathrm{H}$, Igarashi $\mathrm{S}$, Fujimuro $\mathrm{M}$, Agrawal $\mathrm{N}$, Taya $\mathrm{Y}$ et al. p53 mediates cellular dysfunction and behavioral abnormalities in Huntington's disease. Neuron 2005 47: $29-41$.

4. Culmsee C, Mattson MP. p53 in neuronal apoptosis. Biochem Biophys Res Commun 2005; 331: $761-777$.

5. Duan W, Zhu X, Ladenheim B, Yu QS, Guo Z, Oyler J et al. p53 inhibitors preserve dopamine neurons and motor function in experimental parkinsonism. Ann Neurol 2002; 52 597-606.

6. Culmsee C, Zhu X, Yu QS, Chan SL, Camandola S, Guo Z et al. A synthetic inhibitor of p53 protects neurons against death induced by ischemic and excitotoxic insults, and amyloid beta-peptide. J Neurochem 2001; 77: 220-228.

7. Leker RR, Aharonowiz M, Greig NH, Ovadia H. The role of p53-induced apoptosis in cerebral ischemia: effects of the p53 inhibitor pifithrin alpha. Exp Neurol 2004; 187: 478 486

8. Chao C, Saito S, Kang J, Anderson CW, Appella E, Xu Y. p53 transcriptional activity is essential for p53-dependent apoptosis following DNA damage. EMBO J 2000; 19: 49674975.

9. Johnson TM, Hammond EM, Giaccia A, Attardi LD. The p53QS transactivation-deficient mutant shows stress-specific apoptotic activity and induces embryonic lethality. Nat Genet 2005; 37: 145-152.

10. Ihrie RA, Reczek E, Horner JS, Khachatrian L, Sage J, Jacks T et al. Perp is a mediator of p53-dependent apoptosis in diverse cell types. Curr Biol 2003: 13: 1985-1990.

11. Jeffers JR, Parganas E, Lee $\mathrm{Y}$, Yang $C$, Wang J, Brennan $\mathrm{J}$ et al. Puma is an essential mediator of p53-dependent and -independent apoptotic pathways. Cancer Cell 2003; 4 321-328.

12. McCurrach ME, Connor TM, Knudson CM, Korsmeyer SJ, Lowe SW. Bax-deficiency promotes drug resistance and oncogenic transformation by attenuating p53-dependent apoptosis. Proc Natl Acad Sci USA 1997; 94: 2345-2349.

13. Shibue T, Takeda K, Oda E, Tanaka H, Murasawa H, Takaoka A et al. Integral role of Noxa in p53-mediated apoptotic response. Genes Dev 2003; 17: 2233-2238.

14. Villunger A, Michalak EM, Coultas L, Mullauer F, Bock G, Ausserlechner MJ et al. p53- and drug-induced apoptotic responses mediated by $\mathrm{BH} 3-$ only proteins puma and noxa. Science 2003; 302: 1036-1038.

15. Attardi LD, Reczek EE, Cosmas C, Demicco EG, McCurrach ME, Lowe SW et al. PERP, an apoptosis-associated target of $\mathrm{p53}$, is a novel member of the PMP-22/gas3 family. Genes Dev 2000; 14: 704-718.

16. Lowe SW, Ruley HE. Stabilization of the p53 tumor suppressor is induced by adenovirus 5 E1A and accompanies apoptosis. Genes Dev 1993; 7: 535-545.

17. Debbas $M$, White $E$. Wild-type $p 53$ mediates apoptosis by $E 1 A$, which is inhibited by $E 1 B$. Genes Dev 1993; 7: 546-554

18. Soengas MS, Alarcon RM, Yoshida H, Giaccia AJ, Hakem R, Mak TW et al. Apaf-1 and caspase-9 in p53-dependent apoptosis and tumor inhibition. Science 1999; 284: 156-159.

19. Prasad KV, Ao Z, Yoon Y, Wu MX, Rizk M, Jacquot S et al. CD27, a member of the tumor necrosis factor receptor family, induces apoptosis and binds to Siva, a proapoptotic protein Proc Natl Acad Sci USA 1997; 94: 6346-6351. 
20. Masse K, Dabernat S, Bourbon PM, Larou M, Amrein L, Barraud P et al. Characterization of the nm23-M2, nm23-M3 and nm23-M4 mouse genes: comparison with their human orthologs. Gene 2002; 296: 87-97.

21. Mikesch JH, Buerger $H$, Simon R, Brandt B. Decay-accelerating factor (CD55): a versatile acting molecule in human malignancies. Biochim Biophys Acta 2006; 1766: 42-52.

22. Fortin A, MacLaurin JG, Arbour N, Cregan SP, Kushwaha N, Callaghan SM et al. The proapoptotic gene SIVA is a direct transcriptional target for the tumor suppressors p53 and E2F1. J Biol Chem 2004; 279: 28706-28714.

23. Yonish-Rouach E, Resnitzky D, Lotem J, Sachs L, Kimchi A, Oren M. Wild-type p53 induces apoptosis of myeloid leukaemic cells that is inhibited by interleukin-6. Nature 1991; 352: $345-347$.

24. Enokido $\mathrm{Y}$, Araki T, Tanaka K, Aizawa S, Hatanaka H. Involvement of p53 in DNA strand break-induced apoptosis in postmitotic CNS neurons. Eur J Neurosci 1996; 8: 1812-1821.

25. Cory S, Adams JM. The Bcl2 family: regulators of the cellular life-or-death switch. Nat Rev Cancer 2002; 2: 647-656.

26. Py B, Slomianny C, Auberger P, Petit PX, Benichou S. Siva-1 and an alternative splice form lacking the death domain, Siva-2, similarly induce apoptosis in T lymphocytes via caspase-dependent mitochondrial pathway. J Immunol 2004; 172: 4008-4017.

27. Xue L, Chu F, Cheng Y, Sun X, Borthakur A, Ramarao M et al. Siva- 1 binds to and inhibits $\mathrm{BCL}-\mathrm{X}(\mathrm{L})$-mediated protection against UV radiation-induced apoptosis. Proc Natl Acad Sci USA 2002; 99: 6925-6930.

28. Herzog KH, Chong MJ, Kapsetaki M, Morgan JI, McKinnon PJ. Requirement for Atm in ionizing radiation-induced cell death in the developing central nervous system. Science 1998; 280: 1089-1091.

29. Plesnila N, Zinkel S, Le DA, Amin-Hanjani S, Wu Y, Qiu J et al. BID mediates neuronal cell death after oxygen/glucose deprivation and focal cerebral ischemia. Proc Natl Acad Sci USA 2001; 98: 15318-15323.

30. Fortin A, Cregan SP, MacLaurin JG, Kushwaha N, Hickman ES, Thompson CS et al. APAF1 is a key transcriptional target for $p 53$ in the regulation of neuronal cell death. $J$ Cell Biol 2001; 155: 207-216.

31. Xiang H, Kinoshita Y, Knudson CM, Korsmeyer SJ, Schwartzkroin PA, Morrison RS. Bax involvement in p53-mediated neuronal cell death. J Neurosci 1998; 18: 1363-1373.
32. Spinicelli S, Nocentini G, Ronchetti S, Krausz LT, Bianchini R, Riccardi C. GITR interacts with the pro-apoptotic protein Siva and induces apoptosis. Cell Death Differ 2002; 9: 13821384.

33. Chu F, Barkinge J, Hawkins S, Gudi R, Salgia R, Kanteti PV. Expression of Siva-1 protein or its putative amphipathic helical region enhances cisplatin-induced apoptosis in breast cancer cells: effect of elevated levels of BCL-2. Cancer Res 2005; 65: 5301-5309.

34. Semont A, Nowak EB, Silva Lages C, Mathieu C, Mouthon MA, May E et al. Involvement of p53 and Fas/CD95 in murine neural progenitor cell response to ionizing irradiation. Oncogene 2004; 23: 8497-8508.

35. Finnberg N, Gruber JJ, Fei P, Rudolph D, Bric A, Kim SH et al. DR5 knockout mice are compromised in radiation-induced apoptosis. Mol Cell Biol 2005; 25: 2000-2013.

36. Michalak E, Villunger A, Erlacher M, Strasser A. Death squads enlisted by the tumour suppressor p53. Biochem Biophys Res Commun 2005; 331: 786-798.

37. Yin XM, Wang K, Gross A, Zhao Y, Zinkel S, Klocke B et al. Bid-deficient mice are resistant to Fas-induced hepatocellular apoptosis. Nature 1999; 400: 886-891.

38. Mattson MP. Apoptosis in neurodegenerative disorders. Nat Rev Mol Cell Biol 2000; 1 : 120-129.

39. Chung $\mathrm{CH}$, Parker JS, Karaca G, Wu J, Funkhouser WK, Moore D et al. Molecular classification of head and neck squamous cell carcinomas using patterns of gene expression. Cancer Cell 2004; 5: 489-500.

40. Attardi LD, Lowe SW, Brugarolas J, Jacks T. Transcriptional activation by p53, but not induction of the p21 gene, is essential for oncogene-mediated apoptosis. EMBO J 1996; 15: 3693-3701.

41. Bonni A, Brunet A, West AE, Datta SR, Takasu MA, Greenberg ME. Cell survival promoted by the Ras-MAPK signaling pathway by transcription-dependent and -independent mechanisms. Science 1999; 286: 1358-1362.

42. Rubinson DA, Dillon CP, Kwiatkowski AV, Sievers C, Yang L, Kopinja J et al. A lentivirusbased system to functionally silence genes in primary mammalian cells, stem cells and transgenic mice by RNA interference. Nat Genet 2003; 33: 401-406.

43. Vogelmann R, Nelson WJ. Fractionation of the epithelial apical junctional complex: reassessment of protein distributions in different substructures. Mol Biol Cell 2005; 16 : $701-716$.

\section{Supplementary Information accompanies the paper on Cell Death and Differentiation website (http://www.nature.com/cdd)}

